SCIENCE 305 (5688): 1238-1239 AUG 272004

[DOI: 10.1126/science.305.5688.1238c]

http://www.sciencemag.org

\title{
Extending Life-Span in C. elegans
}

\section{Houthoofd K, Braeckman BP, Johnson TE, Vanfleteren JR}

To whom correspondence should be addressed: Jacques.Vanfleteren@UGent.be

The life-span of the nematode Caenorhabditis elegans can be extended by at least six different mechanisms, including calorie restriction, reduced Ins/IGF-1 signaling, germline ablation, food sensing amphid ablation, mitochondrial deficiency, and decreased temperature. Reduced Ins/IGF-1 signaling and calorie restriction can also increase the life-span of flies and mice. The Brevia "Healthy animals with extreme longevity" by $\mathrm{N}$. Arantes-Oliveira et al. (24 Oct. 2003, p. 611) showed that daf-2 RNAi treatment and gonad ablation of worms carrying the daf-2(e1368) hypomorphic mutation in the gene encoding the $C$. elegans Ins/IGF-1 receptor increases their life-span 6.0-fold. We have found that the average life-span of daf-2(e1370) mutants grown in axenic medium [a sterile liquid medium based on yeast extract, soy peptone, and hemoglobin; see (1)] was 90.9 days, representing a 6.3-fold life extension and a 7.5-fold adult life-span extension relative to wild-type controls grown on plate cultures seeded with live E. coli cells (1)).

Arantes-Oliveira et al. also note the health of their long-lived worms. We observed that worms grown in axenic medium appear more vigorous than their monoxenically grown counterparts and that these worms exhibit an increase in metabolic rate (2)), counter to the idea that a reduction of the metabolic rate is associated with a longer life-span. Moreover, both caloric restriction and reduced Ins/IGF-1 signaling increase the resistance to heat and oxidative stressors ( 1 ), and calorically restricted mice are less prone to age-related diseases. Thus, the life of worms can be extended without diminishing health. These results might be important for human aging as well, because both caloric restriction and cell signaling have been shown to regulate the aging rate in organisms ranging from yeast to mammals. 


\title{
References and Notes
}

1. K. Houthoofd, B. P. Braeckman, T. E. Johnson, J. R. Vanfleteren, Exp. Gerontol. 38, 947 (2003).

2. K. Houthoofd et al., Exp. Gerontol. 37, 1371 (2002).

3. K.H. and B.P.B are postdoctoral fellows with the Fund for Scientific ResearchFlanders, Belgium. This research was supported by grants from Ghent University and the Fund for Scientific Research-Flanders (J.V.) and from the U.S. National Institutes for Health and the Ellison Medical Foundation (T.E.J.).

\section{Author info}

\section{Koen Houthoofd,}

Department of Biology,

Ghent University,

K. L. Ledeganckstraat 35,

B-9000 Ghent,

Belgium.

\section{Bart P. Braeckman,}

Department of Biology,

Ghent University,

K. L. Ledeganckstraat 35,

B-9000 Ghent,

Belgium.

\section{Thomas E. Johnson,}

Institute for Behavioral Genetics, University of Colorado at Boulder,

Campus Box 447,

Boulder, CO 80309-0447,

USA.

\author{
Jacques R. Vanfleteren ${ }^{*}$ \\ Department of Biology, \\ Ghent University, \\ K. L. Ledeganckstraat 35, \\ B-9000 Ghent, \\ Belgium.
}

